\title{
Rückenschmerzen: Frühe Krankengymnastik mit nur marginalem Effekt
}

\author{
Ist eine früh einsetzende Krankengymnastik bei Patienten mit neu aufgetrete- \\ nen lumbalen Rückenschmerzen wirksam?
}

Dises ie Lebenszeitprävalenz von akuten Rückenschmerzen beträgt $70 \%$ und $25 \%$ aller Erwachsenen berichten über Rückenschmerzen in den letzten drei Monaten. Rückenschmerzen sind für etwa 3-5\% aller Arztbesuche verantwortlich. Die meisten Patienten werden vom Hausarzt behandelt. Bei chronischen Rückenschmerzen ist der Nutzen von Krankengymnastik und Physiotherapie belegt. Für die Akutsituation gibt es bisher relativ wenige Daten.

Es handelt sich um eine randomisierte klinische Studie mit 220 Teilnehmern. Einschlusskriterien waren akute Rückenschmerzen in den letzten 16 Tagen, Alter zwischen 18 und 60 Jahre und ein Wert auf der Oswestry Disability Index Skala von 20 oder höher. Patienten mit radikulären Schmerzen wurden ausgeschlossen. Alle Patienten erhielten Instruktionen wie sie sich bezüglich der Rückenschmerzen verhalten sollten. Bei der Hälfte der Patienten wurden vier Sitzungen einer Physiotherapie durchgeführt. Diese begann üblicherweise 72 Stunden nach dem Erstkontakt mit dem Arzt. Die Physiotherapie erfasste unter anderem Manualtherapie und Bewegungsübungen. Außerdem wurden den Pati- enten Übungen zur Kräftigung der Muskulatur gezeigt.

Der primäre Outcome war die Veränderung auf der Ostwestry Disability Index Skala nach vier Wochen und nach einem Jahr. Außerdem wurden die Reduktion der Schmerzintensität, die Lebensqualität und die Inanspruchnahme des Gesundheitssystems untersucht. In die übliche Therapie wurden 112 Patienten randomisiert und in die frühe Physiotherapie 108. Die Patienten waren im Mittel 36 Jahre alt und $50 \%$ waren Frauen. Zwei Drittel der Patienten nahmen nicht steroidale Antirheumatika (NSAR) und ein Drittel Opioide ein. Zwei Drittel der Patienten hatten in der Vergangenheit unter Rückenschmerzen gelitten. Die mittlere Schmerzintensität auf einer Skala von 0 bis 10 wurde mit 5 angegeben. Der Wert auf der Oswestry Disability Index Skala lag bei 40 . Nach drei Monaten fand sich ein signifikanter Unterschied zugunsten der Physiotherapie für die allgemeine Behinderung und nach vier Wochen für den Oswestry Disability Index Score. Unterschiede in der Schmerzintensität ließen sich nicht nachweisen. Die Inanspruchnahme des Gesundheitssystems war ebenfalls nicht unterschiedlich.
Fazit: Eine frühe Physiotherapie bei Patienten mit akuten lumbalen Rückenschmerzen führt zu einer geringen Verbesserung der Behinderung und der Schmerzintensität.

Fritz JM, Magel JS, McFadden M et al. Early physical therapy vs usual care in patients with recent-onset low back pain: A randomized clinical trial. JAMA 2015; 314: 1459-67

\section{Kommentar von Prof. Dr. med.}

\section{Hans-Christoph Diener}

Diese prospektive randomisierte Studie zeigt, dass der frühe Einsatz einer Physiotherapie bei Patienten mit akuten Rückenschmerzen zu einer geringeren Besserung von Beschwerden und Behinderungen nach vier Wochen führt. Hierbei muss berücksichtigt werden, dass alle Patienten medikamentös behandelt wurden. Für den klinischen Alltag bedeutet dies, dass zunächst bei Patienten mit akuten Rückenschmerzen der Verlauf unter medikamentöser Therapie mit nicht steroidalen Antirheumatika und bei starken Schmerzen mit retardierten Opioiden abgewartet werden kann. Bestehen die Schmerzen länger als 10 bis 14 Tage, sollten dann allerdings Physiotherapie und Krankengymnastik zum Einsatz kommen.

Prof. Dr. med. Hans-Christoph Diener

\section{Morphin plus Lidocain: Starke Kombi bei Nierenkoliken}

Der extreme Schmerz einer Nierenkolik erfordert eine rasche und effektive Schmerzlinderung, etwa mit Morphin. Kombiniert mit Lidocain ist die Wirkung noch besser.

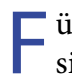
ür eine rasche und effektive Analgesie werden bei Nierenkoliken neben nicht steroidalen Antiphlogistika auch Opioide eingesetzt. Mit der Opioiddosis steigt aber auch das Risiko für substanztypische Nebenwirkungen wie Atemdepression, Übelkeit, Erbrechen und Hypotension. Um die Dosis und somit das Nebenwirkungsrisiko so gering wie möglich halten zu können, gleichzeitig aber keine Abstriche in der Schmerzlinderung hinnehmen zu müssen, haben iranische Anästhesisten Morphin mit Lidocain kombiniert und das analgetische Potenzial mit dem einer alleinigen Morphintherapie randomisiert und doppelblind verglichen. Überprüft haben sie das Therapieregime an 89 Erwachsenen, die zwischen Dezember 2012 und Juli 2013 mit Nierenkoliken im Imam-Khomeini-Krankenhaus in Sari vorstellig wurden. In der Auswertung berücksichtigten sie nur Patienten, bei denen die anfängliche Verdachtsdiagnose über den Nachweis einer Hämaturie oder sonografischer beziehungsweise radiologischer Darstellung des Harnsteines bestätigt werden konnte.

Gruppe A behandelten sie mit 0,1 mg/ $\mathrm{kg}$ Morphin plus $1,5 \mathrm{mg} / \mathrm{kg}$ Lidocain, Gruppe B mit 0,1 mg/kg Morphin plus $9 \%$-iger Kochsalzlösung. Beides verabreichten sie als Bolusinfusion. Vor der Infusion sowie 5, 10, 30, 60 und $120 \mathrm{Mi}-$ nuten nach der Behandlung sollten die Probanden die Schmerzintensität an- 